Eur. J. Math. Anal. 2 (2022) 9

doi: 10.28924/ada/ma.2.9

\title{
Efficient Numerical Schemes for Computations of European Options with Transaction Costs
}

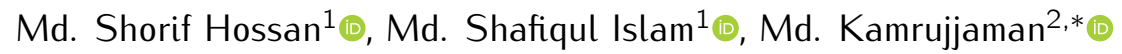 \\ ${ }^{1}$ Department of Applied Mathematics, University of Dhaka, Dhaka, Bangladesh \\ shorif@du.ac.bd, mdshafiqul@du.ac.bd \\ ${ }^{2}$ Department of Mathematics, University of Dhaka, Dhaka, Bangladesh \\ kamrujjaman@du.ac.bd \\ *Correspondence: kamrujjaman@du.ac.bd
}

\begin{abstract}
AвStRACT. This paper aims to find numerical solutions of the non-linear Black-Scholes partial differential equation (PDE), which often appears in financial markets, for European option pricing in the appearance of the transaction costs. Here we exploit the transformations for the computational purpose of a non-linear Black-Scholes PDE to modify as a non-linear parabolic type PDE with reliable initial and boundary conditions for call and put options. Several schemes are derived rigorously using the finite volume method (FVM) and finite difference method (FDM), which is the novelty of this paper. Stability and consistency analysis assure the convergence of these schemes. We apply these schemes to various volatility models, such as the Leland, Boyle and Vorst, Barles and Soner, and Risk-adjusted pricing methodology (RAPM). All the schemes are tested numerically. The convergence of the obtained results is observed, and we find that they are also reliable. Finally, we display all the approximate results together with the exact values through graphical and tabular representations.
\end{abstract}

\section{INTRODUCTION}

Understanding and accurately evaluating transaction costs in a financial market is vital for security trading, asset pricing, stock market regulation, and many other issues. During the last few decades, pricing options more accurately after including realistic assumptions-such as transaction cost, getting more importance from both the traders and the investors.

The literature's [1-6], contains descriptive discussions of options. Fischer Black and Myron Scholes [7] worked jointly, and first disclosed the concept of the Black-Scholes model for options pricing and corporate liabilities, and was published in 1973, while Robert Merton [8] advanced this model in the article "Theory of rational option pricing" in the same year. Their derived equation is based on the assumption that there are no fees for buying and selling options and stocks, as well as no trade barriers (i.e., no commissions and transaction costs). In other words, this model makes

Received: 20 Dec 2021.

Key words and phrases. nonlinear Black-Scholes PDE; option pricing; volatility model; finite volume method; finite difference method. 
a friction-less assumption (which is indispensable, as actual costs correlated with practical market applications) to implement a hedging plan for any contingent claim of the European type.

Various studies have been conducted about the linear Black-Scholes model [9-15] though it adopts the unrealistic assumption of no transaction costs. Several studies have been attempted to evaluate the price of European options [16-23], American options [24-28], Asian options [29, 30], and Barrier options [31] in a completely friction-less market. Recently, the fractional Black-Scholes model [32-34] received some attention.

Contrastingly, the non-linear Black-Scholes PDE, where the non-linear term denotes the presence of transaction costs, is of great importance to our contemporary world over some time both in terms of approach and applicability. Several models [35] consider transaction costs: Leland model, Paras, and Avellaneda model, Boyle and Vorst model, Hodges and Neuberger model, Barles and Soner model, and RAPM (Risk-adjusted pricing methodology) model. If the transaction cost parameters are equal to zero, all of these non-linear transaction cost models are unvarying with the linear model.

Soner et al. [36] showed that there is no nontrivial hedging portfolio for option pricing with transaction costs. They also suggested that the best hedging strategy is buying an asset and taking on it for a certain period as a call or put option. Leland [37] inaugurates the idea of using transaction costs at discrete times. He also indicated that the hedging error could be minimized if the length of re-balancing frequency approaches zero. Later, Boyle and Vorst [38] demonstrated further in a discrete-time framework with a binomial tree model for the option prices with proportional transaction costs, and it is pretty accurate for possible parameter values. Besides, Dewynne et al. [39] considered path-dependent and exotic options with transaction costs. Recently, asymptotic analysis [40] and Markov chain approximation [41] were also studied for pricing European options with transaction costs in some previous literature.

On the other hand, few researchers [42-46,49-51] paid their attention to solve the non-linear Black-Scholes equation numerically. For example, the exponential time differencing (ETD) method [44] was applied to solve the non-linear Black-Scholes model for pricing American options with a highly stable and efficient transaction cost. Lesmana and Wang [45] developed the numerical method based on an upwind finite difference scheme for a non-linear parabolic PDE, and they attempted to pricing European options under transaction costs. Ankudinova and Ehrhardt [46] focused on the non-linear Black-Scholes equation for European call options using several transaction cost models as well as Crank-Nicolson and Rigal compact schemes. R. L. Valkov [47] has solved the non-linear Black-Scholes-Bellman model numerically as well as discuss the monotonicity and consistency of his suggested scheme in considerable detail. A monotone finite volume spatial discretization and a second-order predictor-corrector scheme in time are considered by Radoslas Valkov [48] to handle the Black-Scholes equation with uncertain volatility and dividend. The applicability of implicit 
numerical schemes for the valuation of contingent claims in non-linear Black-Scholes models has been discussed by Pascal Heider [49]. He also studied the practical implications of the derived stability criteria on relevant numerical examples. He claimed that if certain stability requirements are satisfied, it is possible to construct convergent implicit algorithms for non-linear Black-Scholes equations. Ekaterina Dremkova and Matthias Ehrhardt [50] have solved non-linear Black-Scholes equations for American options with a non-linear volatility function using various compact finite difference techniques to improve the order of the accuracy. The existence and uniqueness of solutions to the well-known non-linear Black-Scholes equation have been demonstrated by Naoyuki Ishimura [51] for both in the classical and weak senses.

However, in this paper, we work on approximating non-linear Black-Scholes PDE for valuing European options when there are transaction costs. For this, we organize the present research work as follows: we modify the original model into parabolic type PDE exploiting the transformations [46] which are written in section 2. A brief description of different volatility models is given in section 3 subsequently. Section 4 is devoted to discretize the transformed parabolic type equation by using some numerical schemes. Stability and consistency analysis are included in sections 5 and 6 , respectively. In section 7, numerical examples are given to show the efficacy of the proposed schemes. Subsequently, a general conclusion is drawn in section 8. Finally, all relevant references are included.

\section{The Model Equation}

This section considers a non-linear Black-Scholes PDE and modifies it to a non-linear parabolic type equation with appropriate and available transformations, which would be easy to compute numerically. Let us consider the non-linear Black-Scholes PDE [46],

$$
\frac{\partial F}{\partial t}+r S \frac{\partial F}{\partial S}+\frac{1}{2} \tilde{\sigma}^{2} S^{2} \frac{\partial^{2} F}{\partial S^{2}}-r F=0,0<S<\infty, t \in(0, T)
$$

subject to the terminal and boundary conditions for European call and put options:

$F(S, T)=\max (S-K, 0), F(S, t)=0$ when $S=0, F(S, t)=S-K e^{-r(T-t)}$, when $S \rightarrow \infty$ and $F(S, T)=\max (K-S, 0), F(S, t)=K e^{-r(T-t)}$, when $S=0, F(S, t)=0$, when $S \rightarrow \infty$ respectively. Throughout this paper, we use the notations: $F=F(S, t)=$ the option price, $S=$ stock price, $K=$ strike price, $T=$ maturity time, $r=$ interest rate, $t=$ time in years, and $\tilde{\sigma}=\tilde{\sigma}\left(t, S, \frac{\partial F}{\partial S}, \frac{\partial^{2} F}{\partial S^{2}}\right)$ depends on the volatility model.

Now consider the transformations [46] as given below,

$$
y=\ln \left(K^{-1} S\right), \tau=\frac{1}{2} \sigma^{2}(T-t) \text { and } u(y, t)=K^{-1} e^{-y} F(S, t)
$$

and substituting these into Equation (1) to obtain the following non-linear parabolic PDE 


$$
\frac{\partial u}{\partial \tau}=\frac{2 r}{\sigma^{2}} \frac{\partial u}{\partial y}+\left(\frac{\tilde{\sigma}}{\sigma}\right)^{2}\left(\frac{\partial^{2} u}{\partial y^{2}}+\frac{\partial u}{\partial y}\right), y_{\min }<y<y_{\max }, \tau \in\left(0, \frac{\sigma^{2}}{2} T\right)
$$

with the modified initial and boundary conditions for European call and put options:

$u(y, 0)=\max \left(1-e^{-y}, 0\right)$ as $y \in(-\infty, \infty), u(y, \tau)=0$ as $y \rightarrow-\infty, u(y, \tau)=1-e^{-\left(y+2 r \tau / \sigma^{2}\right)}$ as $y \rightarrow \infty$, and $u(y, 0)=\max \left(e^{-y}-1,0\right)$ as $y \in(-\infty, \infty), u(y, \tau)=e^{-\left(y+2 r \tau / \sigma^{2}\right)}$ as $y \rightarrow-\infty$, $u(y, \tau)=0$ as $y \rightarrow \infty$, respectively.

\section{Volatility Models}

This section concerns four stochastic volatility models to discretize the non-linear Black-Scholes PDE, whose solution provides the option price for transaction fees. We give a short description, but details are available in some previous literature [46].

Leland Volatility Model (LVM). Leland [37] developed a technique for replicating options in the presence of transactions costs for a small time interval. He proposed that the option price is the solution of the non-linear Black-Scholes Equation (1) but with the adjusted volatility [46] as follows:

$$
\tilde{\sigma}=\sigma \sqrt{1+\sqrt{\frac{2}{\pi}} \frac{\mu}{\sigma \sqrt{\Delta t}} \operatorname{sign}\left(F_{S S}\right)}
$$

where, $\sigma$ is the original volatility, $\mu$ is the round-trip transaction cost per unit dollar of the transaction, and $\Delta t$ is the transaction frequency. In this formula, both $\mu$ and $\Delta t$ are assumed to be small while keeping the ratio $\frac{\mu}{\sqrt{\Delta t}}$ of order one.

Boyle and Vorst Volatility Model (BVVM). Boyle and Vorst [38] derived a method for calculating option prices in a discrete-time where option price meets to Black-Scholes price with the modified volatility [46] given by

$$
\tilde{\sigma}=\sigma \sqrt{1+\frac{\mu}{\sigma \sqrt{\Delta t}} \operatorname{sign}\left(F_{S S}\right)}
$$

where, $\sigma, \mu$, and $\Delta t$ represents the same meaning as Leland.

Barles and Soner Volatility Model (BSVM). Barles and Soner [43] evolved a model using the utility function approach of Hodges and Neuberger [52] along with asymptotic analysis of partial differential equations. For this case, the formula for the modified volatility [46] is given by

$$
\tilde{\sigma}=\sigma \sqrt{1+e^{r(T-t)} a^{2} S^{2} F_{S S}}
$$

where, $\mu=a \sqrt{\epsilon}$ is the round-trip transaction cost per unit dollar of the transaction for some constant $a>0$ and $\in \rightarrow 0$. 
RAPM Volatility Model (RAPMVM). Kratka [53] took the first step for this model and later improved by Jandačka and Ševčovič [54]. Here the modified volatility is of the form [46]

$$
\tilde{\sigma}=\sigma \sqrt{1+3 \times \sqrt[3]{\frac{C^{2} M}{2 \pi} S F_{S S}}}
$$

where, $M \geq 0$ is the transaction cost measure and $C \geq 0$ is the risk premium measure.

\section{Derivations of Computational Schemes}

In this section, we derive five computational schemes, in detail, for Equation (2) using two well-known numerical methods.

4.1. Dufort-Frankel Finite Difference Scheme. The Dufort-Frankel FD scheme [55] can be applied to solve various kinds of problems which occur in finance. This scheme is a multi-step method, and requires another scheme for simulating the first temporal vector. In this formulation $\frac{\partial u}{\partial \tau}, \frac{\partial u}{\partial y}$, and $\frac{\partial^{2} u}{\partial y^{2}}$ are discretized by central difference and $u_{i}^{j}$ is replaced by $\left(u_{i}^{j+1}+u_{i}^{j-1}\right) / 2$. Thus, discretizing Equation (2) by Dufort-Frankel FDM, we obtain

$$
\begin{aligned}
\frac{1}{2 \Delta \tau}\left(u_{i}^{j+1}-u_{i}^{j-1}\right)= & \left(\frac{\tilde{\sigma}}{\sigma}\right)^{2}\left[\frac{1}{(\Delta y)^{2}}\left(u_{i-1}^{j}-\left(u_{i}^{j+1}+u_{i}^{j-1}\right)+u_{i+1}^{j}\right)\right] \\
& +\left(\frac{\tilde{\sigma}}{\sigma}\right)^{2}\left[\frac{1}{2 \Delta y}\left(u_{i+1}^{j}-u_{i-1}^{j}\right)\right]+\frac{r}{\sigma^{2} \Delta y}\left(u_{i+1}^{j}-u_{i-1}^{j}\right)
\end{aligned}
$$

or, equivalently

$$
\begin{aligned}
u_{i}^{j+1}= & u_{i}^{j-1}+\frac{2 r(\Delta \tau)(\Delta y)}{\sigma^{2}}\left(u_{i+1}^{j}-u_{i-1}^{j}\right)+\frac{\Delta \tau}{\Delta y}\left(\frac{\tilde{\sigma}}{\sigma}\right)^{2}\left(u_{i+1}^{j}-u_{i-1}^{j}\right) \\
& +\frac{2(\Delta \tau)}{(\Delta y)^{2}}\left(\frac{\tilde{\sigma}}{\sigma}\right)^{2}\left(u_{i-1}^{j}-u_{i}^{j+1}-u_{i}^{j-1}+u_{i+1}^{j}\right)
\end{aligned}
$$

which can be written as

$$
u_{i}^{j+1}=a_{i} u_{i-1}^{j}+b_{i} u_{i+1}^{j}+c_{i} u_{i}^{j-1} ; i=0,1,2, \ldots, n-1 ; j=0,1,2, \ldots, m-1
$$

where

$$
\begin{aligned}
& a_{i}=\left[(\Delta y)^{2}+2(\Delta \tau)\left(\frac{\tilde{\sigma}}{\sigma}\right)^{2}\right]^{-1} \times\left[(\Delta \tau)\left(\frac{\tilde{\sigma}}{\sigma}\right)^{2}(2-\Delta y)-(\Delta y)(\Delta \tau) \frac{2 r}{\sigma^{2}}\right], \\
& b_{i}=\left[(\Delta y)^{2}+2(\Delta \tau)\left(\frac{\tilde{\sigma}}{\sigma}\right)^{2}\right]^{-1} \times\left[(\Delta \tau)\left(\frac{\tilde{\sigma}}{\sigma}\right)^{2}(2+\Delta y)+(\Delta y)(\Delta \tau) \frac{2 r}{\sigma^{2}}\right],
\end{aligned}
$$

and

$$
c_{i}=\left[(\Delta y)^{2}+2(\Delta \tau)\left(\frac{\tilde{\sigma}}{\sigma}\right)^{2}\right]^{-1} \times\left[(\Delta y)^{2}-2(\Delta \tau)\left(\frac{\tilde{\sigma}}{\sigma}\right)^{2}\right]
$$

which is our proposed Dufort-Frankel Finite Difference Scheme (DFFDS). 
4.2. Laasonen Finite Difference Scheme. The Laasonen finite difference scheme [55] can be applied to solve linear and non-linear partial differential equations. This method metamorphosed partial differential equations into a system of linear algebraic equations. In this formulation $\frac{\partial u}{\partial \tau}$ is approximated by a central differencing at a step $\frac{\Delta \tau}{2}$, and $\frac{\partial u}{\partial y}, \frac{\partial^{2} u}{\partial y^{2}}$ are approximated by central differences at time levels $j+1$. Now the discretized form of Equation (2) is as follows

$$
\begin{aligned}
\frac{1}{\Delta \tau}\left(u_{i}^{j+1}-u_{i}^{j}\right)= & \frac{1}{2(\Delta y)^{2}}\left(\frac{\tilde{\sigma}}{\sigma}\right)^{2}\left[2\left(u_{i-1}^{j+1}-2 u_{i}^{j+1}+u_{i+1}^{j+1}\right)+\Delta y\left(u_{i+1}^{j+1}-u_{i-1}^{j+1}\right)\right] \\
& +\frac{r}{\sigma^{2} \Delta y}\left(u_{i+1}^{j+1}-u_{i-1}^{j+1}\right)
\end{aligned}
$$

After simplification, we get

$$
\begin{gathered}
{\left[\frac{r \Delta \tau}{\Delta y \sigma^{2}}+\frac{\Delta \tau}{2(\Delta y)^{2}}\left(\frac{\tilde{\sigma}}{\sigma}\right)^{2}(\Delta y-2)\right] u_{i-1}^{j+1}+\left[1+\frac{2 \Delta \tau}{(\Delta y)^{2}}\left(\frac{\tilde{\sigma}}{\sigma}\right)^{2}\right] u_{i}^{j+1}} \\
-\left[\frac{r \Delta \tau}{\Delta y \sigma^{2}}+\frac{\Delta \tau}{2(\Delta y)^{2}}\left(\frac{\tilde{\sigma}}{\sigma}\right)^{2}(\Delta y+2)\right] u_{i+1}^{j+1}=u_{i}^{j}
\end{gathered}
$$

The above equation reduces to

$$
d_{i} u_{i-1}^{j+1}+\left(1+e_{i}\right) u_{i}^{j+1}+f_{i} u_{i+1}^{j+1}=u_{i}^{j} ; i=0,1,2, \ldots, n-1 ; j=0,1,2, \ldots, m-1
$$

where

$$
d_{i}=\frac{r \Delta \tau}{\Delta y \sigma^{2}}+\frac{\Delta \tau}{2(\Delta y)^{2}}\left(\frac{\tilde{\sigma}}{\sigma}\right)^{2}(\Delta y-2), e_{i}=\frac{2 \Delta \tau}{(\Delta y)^{2}}\left(\frac{\tilde{\sigma}}{\sigma}\right)^{2}
$$

and

$$
f_{i}=-\frac{r \Delta \tau}{\Delta y \sigma^{2}}-\frac{\Delta \tau}{2(\Delta y)^{2}}\left(\frac{\tilde{\sigma}}{\sigma}\right)^{2}(\Delta y+2)
$$

4.3. Finite Volume Schemes. The finite volume scheme is a scheme of solving different kinds of time-dependent or independent partial differential equations in algebraic equations. In this scheme, we divide the physical space into a finite number of control volumes. In this section, we describe it in a few lines, but details are available in the previous study [56] conducted by Malalasekera et al.

Applying the finite volume integration in Equation (2) over a control volume (CV) with a finite time step $\Delta \tau$, we obtain

$$
\begin{aligned}
\int_{\tau}^{\tau+\Delta \tau} \int_{C V} \frac{\partial u}{\partial \tau} d V d \tau=\left(\frac{2 r}{\sigma^{2}}\right. & \left.+\left(\frac{\tilde{\sigma}}{\sigma}\right)^{2}\right) \int_{\tau}^{\tau+\Delta \tau} \int_{C V} \frac{\partial u}{\partial y} d V d \tau \\
& +\left(\frac{\tilde{\sigma}}{\sigma}\right)^{2} \int_{\tau}^{\tau+\Delta \tau} \int_{C V} \frac{\partial^{2} u}{\partial y^{2}} d V d \tau
\end{aligned}
$$


After rearranging, we get

$$
\begin{aligned}
\int_{C V}\left[\int_{\tau}^{\tau+\Delta \tau} \frac{\partial u}{\partial \tau} d \tau\right] d V=\left(\frac{2 r}{\sigma^{2}}\right. & \left.+\left(\frac{\tilde{\sigma}}{\sigma}\right)^{2}\right) \int_{\tau}^{\tau+\Delta \tau}\left[\int_{C V} \frac{\partial u}{\partial y} d V\right] d \tau \\
& +\left(\frac{\tilde{\sigma}}{\sigma}\right)^{2} \int_{\tau}^{\tau+\Delta}\left[\int_{C V} \frac{\partial^{2} u}{\partial y^{2}} d V\right] d \tau
\end{aligned}
$$

Applying Gauss's divergence theorem, the above equation leads

$$
\begin{aligned}
\left(u_{P}-u_{P}^{0}\right) \Delta V= & \frac{1}{2}\left(\frac{2 r}{\sigma^{2}}+\left(\frac{\tilde{\sigma}}{\sigma}\right)^{2}\right) A \int_{\tau}^{\tau+\Delta \tau}\left(u_{E}-u_{W}\right) d \tau \\
& +\left(\frac{\tilde{\sigma}}{\sigma}\right)^{2} \int_{\tau}^{\tau+\Delta \tau}\left[\left(A \frac{u_{E}-u_{P}}{\delta y_{P E}}\right)-\left[\left(A \frac{u_{P}-u_{W}}{\delta y_{W P}}\right)\right]\right] d \tau
\end{aligned}
$$

For $0 \leq \theta \leq 1$, we assume

$$
\int_{\tau}^{\tau+\Delta \tau} u_{P} d \tau=\left[\theta u_{P}+(1-\theta) u_{P}^{0}\right] \Delta \tau
$$

Applying Equation (10) into Equation (9) and dividing by we get

$$
\begin{aligned}
\left(u_{P}-u_{P}^{0}\right) \frac{\Delta y}{\Delta \tau}= & \frac{1}{2}\left(\frac{2 r}{\sigma^{2}}+\left(\frac{\tilde{\sigma}}{\sigma}\right)^{2}\right)\left[\theta\left(u_{E}-u_{W}\right)+(1-\theta)\left(u_{E}^{0}-u_{W}^{0}\right)\right] \\
& +\left(\frac{\tilde{\sigma}}{\sigma}\right)^{2} \theta\left(\frac{u_{E}-u_{P}}{\delta y_{P E}}-\frac{u_{P}-u_{W}}{\delta y_{W P}}\right) \\
& +\left(\frac{\tilde{\sigma}}{\sigma}\right)^{2}(1-\theta)\left(\frac{u_{E}^{0}-u_{P}^{0}}{\delta y_{P E}}-\frac{u_{P}^{0}-u_{W}^{0}}{\delta y_{W P}}\right)
\end{aligned}
$$

For convenience, we put $\delta y_{W P}=\delta y_{P E}=\Delta y$ on the following three schemes.

Explicit Scheme. Substitution of $\theta=0$ into Equation (11) gives the following explicit discretized equation,

$$
\left(u_{P}-u_{P}^{0}\right) \frac{\Delta y}{\Delta \tau}=\frac{1}{2}\left(\frac{2 r}{\sigma^{2}}+\left(\frac{\tilde{\sigma}}{\sigma}\right)^{2}\right)\left(u_{E}^{0}-u_{W}^{0}\right)+\frac{1}{\Delta y}\left(\frac{\tilde{\sigma}}{\sigma}\right)^{2}\left(u_{E}^{0}-2 u_{P}^{0}+u_{W}^{0}\right)
$$

This equation may be re-writtens as

$$
u_{P}=\alpha_{i} u_{W}^{0}+\left(1+\beta_{i}\right) u_{P}^{0}+\gamma_{i} u_{E}^{0}
$$

where

$$
\alpha_{i}=\frac{\Delta \tau}{(\Delta y)^{2}}\left(\frac{\tilde{\sigma}}{\sigma}\right)^{2}-\frac{\Delta \tau}{2 \Delta y}\left(\frac{2 r}{\sigma^{2}}+\left(\frac{\tilde{\sigma}}{\sigma}\right)^{2}\right), \beta_{i}=-\frac{2 \Delta \tau}{(\Delta y)^{2}}\left(\frac{\tilde{\sigma}}{\sigma}\right)^{2}
$$

and

$$
\gamma_{i}=\frac{\Delta \tau}{(\Delta y)^{2}}\left(\frac{\tilde{\sigma}}{\sigma}\right)^{2}+\frac{\Delta \tau}{2 \Delta y}\left(\frac{2 r}{\sigma^{2}}+\left(\frac{\tilde{\sigma}}{\sigma}\right)^{2}\right)
$$

which is the desired Finite Volume Explicit Scheme (FVES). 
Crank-Nicolson Scheme. Putting $\theta=\frac{1}{2}$ into Equation (11), we get the following Crank-Nicolson discretized equation,

$$
\begin{aligned}
\left(u_{P}-u_{P}^{0}\right) \frac{\Delta y}{\Delta \tau}= & \frac{1}{4}\left(\frac{2 r}{\sigma^{2}}+\left(\frac{\tilde{\sigma}}{\sigma}\right)^{2}\right)\left(u_{E}-u_{W}+u_{E}^{0}-u_{W}^{0}\right) \\
& +\frac{1}{2 \Delta y}\left(\frac{\tilde{\sigma}}{\sigma}\right)^{2}\left(u_{E}-2 u_{P}+u_{W}+u_{E}^{0}-2 u_{P}^{0}+u_{W}^{0}\right)
\end{aligned}
$$

After simplification, we get the following equation

$$
\lambda_{i} u_{w}+\left(1+\xi_{i}\right) u_{P}+\eta_{i} u_{E}=-\lambda_{i} u_{\pi}^{0}+\left(1-\xi_{i}\right) u_{P}^{0}-\eta_{i} u_{E}^{0}
$$

where

$$
\lambda_{i}=\frac{\Delta \tau}{4 \Delta y}\left(\frac{2 r}{\sigma^{2}}+\left(\frac{\tilde{\sigma}}{\sigma}\right)^{2}\right)-\frac{\Delta \tau}{2(\Delta y)^{2}}\left(\frac{\tilde{\sigma}}{\sigma}\right)^{2}, \xi_{i}=\frac{\Delta \tau}{(\Delta y)^{2}}\left(\frac{\tilde{\sigma}}{\sigma}\right)^{2}
$$

and

$$
\eta_{i}=-\left[\frac{\Delta \tau}{4 \Delta y}\left(\frac{2 r}{\sigma^{2}}+\left(\frac{\tilde{\sigma}}{\sigma}\right)^{2}\right)+\frac{\Delta \tau}{2(\Delta y)^{2}}\left(\frac{\tilde{\sigma}}{\sigma}\right)^{2}\right]
$$

which is the proposed Finite Volume Crank-Nicolson Scheme (FVCNS).

Fully Implicit Scheme. Substitution of $\theta=1$ into Equation (11) leads to the following form:

$$
\left(u_{P}-u_{P}^{0}\right) \frac{\Delta y}{\Delta \tau}=\frac{1}{2}\left(\frac{2 r}{\sigma^{2}}+\left(\frac{\tilde{\sigma}}{\sigma}\right)^{2}\right)\left(u_{E}-u_{W}\right)+\frac{1}{\Delta y}\left(\frac{\tilde{\sigma}}{\sigma}\right)^{2}\left(u_{E}-2 u_{P}+u_{W}\right)
$$

and the reduced formula is then

$$
q_{i} u_{W}+\left(1+r_{i}\right) u_{P}+s_{i} u_{E}=u_{P}^{0}
$$

where

$$
q_{i}=\frac{\Delta \tau}{2 \Delta y}\left(\frac{2 r}{\sigma^{2}}+\left(\frac{\tilde{\sigma}}{\sigma}\right)^{2}\right)-\frac{\Delta \tau}{(\Delta y)^{2}}\left(\frac{\tilde{\sigma}}{\sigma}\right)^{2}, r_{i}=\frac{2 \Delta \tau}{(\Delta y)^{2}}\left(\frac{\tilde{\sigma}}{\sigma}\right)^{2}
$$

and

$$
s_{i}=-\frac{\Delta \tau}{2 \Delta y}\left(\frac{2 r}{\sigma^{2}}+\left(\frac{\tilde{\sigma}}{\sigma}\right)^{2}\right)-\frac{\Delta \tau}{(\Delta y)^{2}}\left(\frac{\tilde{\sigma}}{\sigma}\right)^{2}
$$

which is our proposed Finite Volume Fully Implicit Scheme (FVFIS).

\section{Stability OF the NUMERICAL SCHEMES}

To test the stability of the derived schemes in section 4, with the help of the Von-Neumann stability method [55], let us consider a Fourier component for $u_{i}^{j}$ and $u_{P}^{0}$ as

$$
u_{i}^{j}=U^{j} e^{l \theta i} \text { and } u_{P}^{0}=U^{j} e^{I \theta i}
$$

where $I=\sqrt{-1}$, i.e., imaginary unit, $U^{j}$ is the amplitude at a time level $j, \theta(=R \Delta y)$ is the phase angle, $R$ is the wave number in the $x$-direction, and $i$ represents the index of the node. 
Similarly,

$$
\begin{aligned}
& u_{i \pm 1}^{j \mp 1}=U^{j \mp 1} e^{l \theta(i \pm 1)} \quad u_{W}^{0}=U^{j} e^{l \theta(i-1)} \quad u_{E}^{0}=U^{j} e^{l \theta(i+1)} \quad u_{P}=U^{j+1} e^{l \theta i} \\
& u_{W}=U^{j+1} e^{l \theta(i-1)} \quad u_{E}=U^{j+1} e^{l \theta(i+1)}
\end{aligned}
$$

For convenience, let us suppose that $G=\frac{u^{j+1}}{u^{j}}$. Thus, the stability requirement is $|G|^{2} \leq 1$. Applying Equation (15) and Equation (16) into Equation (7), and dividing by $e^{\mid \theta i}$, we get

$$
\begin{aligned}
|G|^{2}=\frac{1}{4}[ & \left.\left\{4 \Delta \tau\left(\frac{\tilde{\sigma}}{\sigma}\right)^{2} \cos \theta \pm \sqrt{A}\right\}^{2}+4(\Delta \tau)^{2}(\Delta y)^{2}\left(\frac{2 r}{\sigma^{2}}+\left(\frac{\tilde{\sigma}}{\sigma}\right)^{2}\right)^{2}\left(1-\cos ^{2} \theta\right)\right] \\
& \times\left((\Delta y)^{2}+2 \Delta \tau\left(\frac{\tilde{\sigma}}{\sigma}\right)^{2}\right)^{-2}
\end{aligned}
$$

where

$$
\begin{aligned}
A= & 16(\Delta \tau)^{2}\left(\frac{\tilde{\sigma}}{\sigma}\right)^{4} \cos ^{2} \theta-4(\Delta \tau)^{2}(\Delta y)^{2}\left(\frac{2 r}{\sigma^{2}}+\left(\frac{\tilde{\sigma}}{\sigma}\right)^{2}\right)^{2}\left(1-\cos ^{2} \theta\right)+4(\Delta y)^{4} \\
& -16(\Delta \tau)^{2}\left(\frac{\tilde{\sigma}}{\sigma}\right)^{4}+I 16(\Delta \tau)^{2}(\Delta y)\left(\frac{\tilde{\sigma}}{\sigma}\right)^{2}\left(\frac{2 r}{\sigma^{2}}+\left(\frac{\tilde{\sigma}}{\sigma}\right)^{2}\right) \cos \theta \sqrt{1-\cos ^{2} \theta}
\end{aligned}
$$

For extremum value of $|G|^{2}$, solving $\frac{d|G|^{2}}{d(\cos \theta)}=0$ for $\cos \theta$, and substituting it into $\frac{d^{2}|G|^{2}}{d(\cos \theta)^{2}}<0$. Then from Equation (17), we cannot confirm that the maximum value of $|G|^{2}$ would occur. However, the extreme values of $\cos \theta$ must yet be investigated. For $\cos \theta=1$, Equation (17) gives $|G|^{2}=1$, and the stability requirement is satisfied. For $\cos \theta=-1$, Equation (17) also yields $|G|^{2}=1$ and, and the stability requirement is satisfied. Thus, the DFFDS proposed in Equation (7) is unconditionally stable.

Similarly, we can show that LFDS and FVFIS wrote in Equation (8) and Equation (14), respectively, both are unconditionally stable.

Again, applying Equation (15) and Equation (16) into Equation (12) and dividing by $e^{\mid \theta i}$, we get

$$
G=1+\frac{2 \Delta \tau}{(\Delta y)^{2}}\left(\frac{\tilde{\sigma}}{\sigma}\right)^{2}(\cos \theta-1)+I \frac{\Delta \tau}{\Delta y}\left(\frac{2 r}{\sigma^{2}}+\left(\frac{\tilde{\sigma}}{\sigma}\right)^{2}\right) \sin \theta
$$

Then we may obtain easily,

$$
|G|^{2}=\left\{1+\frac{2 \Delta \tau}{(\Delta y)^{2}}\left(\frac{\tilde{\sigma}}{\sigma}\right)^{2}(\cos \theta-1)\right\}^{2}+\left(\frac{\Delta \tau}{\Delta y}\right)^{2}\left(\frac{2 r}{\sigma^{2}}+\left(\frac{\tilde{\sigma}}{\sigma}\right)^{2}\right)^{2}\left(1-\cos ^{2} \theta\right)
$$

For extremum value of $|G|^{2}$ such that $\frac{d|G|^{2}}{d(\cos \theta)}=0$, we can find

$$
\cos \theta=\frac{1}{\Delta \tau} \times\left[2\left(\frac{\tilde{\sigma}}{\sigma}\right)^{2}-4 \frac{\Delta \tau}{(\Delta y)^{2}}\left(\frac{\tilde{\sigma}}{\sigma}\right)^{4}\right] \times\left(\left(\frac{2 r}{\sigma^{2}}+\left(\frac{\tilde{\sigma}}{\sigma}\right)^{2}\right)^{2}-4 \frac{\Delta \tau}{(\Delta y)^{2}}\left(\frac{\tilde{\sigma}}{\sigma}\right)^{4}\right)^{-1}
$$


Considering $\frac{d^{2}|G|^{2}}{d(\cos \theta)^{2}}<0$, and substituting the value of $\cos \theta$ from Equation (19) into Equation (18), which does not provide us the maximum value of $|G|^{2}$. But, the extreme values of $\cos \theta$ must be investigated. For $\cos \theta=1$, Equation (18) gives $|G|^{2}=1$, and the stability requirement is satisfied. For $\cos \theta=-1$, Equation (18) yields $|G|^{2}=\left\{1-\frac{4 \Delta \tau}{(\Delta y)^{2}}\left(\frac{\tilde{\sigma}}{\sigma}\right)^{2}\right\}^{2}$ and, imposing the requirement of $|G|^{2} \leq 1$, yields, FVES in Equation (12) is conditionally stable and the condition is

$$
\left(\frac{\tilde{\sigma}}{\sigma}\right)^{2} \leq \frac{(\Delta y)^{2}}{2 \Delta \tau}
$$

Similarly, we can state that FVCNS, Equation (13) is also conditionally stable and the condition is

$$
\left(\frac{\tilde{\sigma}}{\sigma}\right)^{2} \leq \frac{(\Delta y)^{2}}{\Delta \tau}
$$

\section{Consistency of the nUmerical SCHEMES}

For consistency, the finite difference equation (FDE) approximation of a PDE must reduce to the original PDE as the step sizes approach zero [55].

Now expanding each $u(y, \tau)$ in a Taylor series expansion about $u_{i}^{j}$, we get

$$
\begin{aligned}
u_{i}^{j+1}= & u_{i}^{j}+\Delta \tau \frac{\partial u}{\partial \tau}+\frac{(\Delta \tau)^{2}}{2 !} \frac{\partial^{2} u}{\partial \tau^{2}}+\frac{(\Delta \tau)^{3}}{3 !} \frac{\partial^{3} u}{\partial \tau^{3}}+O(\Delta \tau)^{4} \\
u_{i+1}^{j+1}= & u_{i}^{j}+\Delta \tau \frac{\partial u}{\partial \tau}+\Delta y \frac{\partial u}{\partial y}+\frac{1}{2 !}\left(\Delta \tau \frac{\partial}{\partial \tau}+\Delta y \frac{\partial}{\partial y}\right)^{2} u \\
& +\frac{1}{3 !}\left(\Delta \tau \frac{\partial}{\partial \tau}+\Delta y \frac{\partial}{\partial y}\right)^{3} u+O\left[(\Delta \tau)^{4},(\Delta y)^{4}\right] \\
u_{i-1}^{j+1}= & u_{i}^{j}+\Delta \tau \frac{\partial u}{\partial \tau}-\Delta y \frac{\partial u}{\partial y}+\frac{1}{2 !}\left(\Delta \tau \frac{\partial}{\partial \tau}-\Delta y \frac{\partial}{\partial y}\right)^{2} u \\
+ & \frac{1}{3 !}\left(\Delta \tau \frac{\partial}{\partial \tau}-\Delta y \frac{\partial}{\partial y}\right)^{3} u+O\left[(\Delta \tau)^{4},(\Delta y)^{4}\right]
\end{aligned}
$$

Applying Equations (22), (23), and (24) into Equation (8) yields

$$
\begin{aligned}
& \left(d_{i}+e_{i}+f_{i}\right) u_{i}^{j}+\left(1+d_{i}+e_{i}+f_{i}\right) \Delta \tau \frac{\partial u}{\partial \tau}+\left(1+d_{i}+e_{i}+f_{i}\right) \frac{(\Delta \tau)^{2}}{2} \frac{\partial^{2} u}{\partial \tau^{2}} \\
& +\left(-d_{i}+f_{i}\right) \Delta y \frac{\partial u}{\partial y}+\left(-d_{i}+f_{i}\right) \Delta \tau \Delta y \frac{\partial^{2} u}{\partial \tau \partial y}+\left(d_{i}+f_{i}\right) \frac{(\Delta y)^{2}}{2} \frac{\partial^{2} u}{\partial y^{2}} \\
& \quad+O\left[(\Delta \tau)^{3},(\Delta y)^{3}\right]=0
\end{aligned}
$$

from which we get

$$
\begin{aligned}
\frac{\partial u}{\partial \tau}+\frac{\Delta \tau}{2} \frac{\partial^{2} u}{\partial \tau^{2}} & -\left(\frac{2 r}{\sigma^{2}}+\left(\frac{\tilde{\sigma}}{\sigma}\right)^{2}\right) \frac{\partial u}{\partial y}-\Delta \tau\left(\frac{2 r}{\sigma^{2}}+\left(\frac{\tilde{\sigma}}{\sigma}\right)^{2}\right) \frac{\partial^{2} u}{\partial y \partial \tau} \\
& -\left(\frac{\tilde{\sigma}}{\sigma}\right)^{2} \frac{\partial^{2} u}{\partial y^{2}}+O\left[(\Delta \tau)^{2},(\Delta y)^{2}\right]=0
\end{aligned}
$$


It is obvious that if $\Delta \tau, \Delta y \rightarrow 0$, then the original PDE (2) is recovered. Therefore, the Laasonen finite difference scheme, Equation (8), is consistent. Now according to Lax's equivalence theorem, [55], LFDS is convergent for all values of the parameters. Similar arguments hold for DFFDS and FVFIS. On the other hand, FVES and FVCNS are also convergent if the conditions (20) and (21) respectively, are satisfied.

TABLE 1. Call option prices using the Leland volatility model.

\begin{tabular}{c|c|c|c|c|c|c}
\hline \multirow{2}{*}{$S_{0}$} & \multirow{2}{*}{$\begin{array}{c}\text { Exact } \\
\text { (Linear) }\end{array}$} & \multicolumn{2}{|l|}{ Finite Difference Schemes } & \multicolumn{3}{|c}{ Finite Volume Schemes } \\
\cline { 3 - 7 } & DFFDS & LFDS & FVES & FVFIS & FVCNS \\
\hline 37.00 & 0.00001 & 0.04734 & 0.04893 & 0.00000 & 0.00054 & 0.00050 \\
\hline 47.00 & 0.00182 & 0.30914 & 0.31368 & 0.00006 & 0.01340 & 0.01297 \\
\hline 57.00 & 0.05078 & 1.09349 & 1.09949 & 0.00036 & 0.10771 & 0.10588 \\
\hline 67.00 & 0.45226 & 2.93576 & 2.94472 & 0.00335 & 0.65191 & 0.64795 \\
\hline 77.00 & 1.97686 & 6.06630 & 6.07104 & 0.01709 & 2.26632 & 2.26230 \\
\hline 87.00 & 5.46222 & 10.56460 & 10.56947 & 1.25649 & 5.63768 & 5.63688 \\
\hline 97.00 & 11.17037 & 16.34912 & 16.34757 & 6.49278 & 11.07370 & 11.07714 \\
\hline 107.00 & 18.71972 & 23.33442 & 23.33541 & 16.15278 & 18.66210 & 18.66616 \\
\hline 117.00 & 27.48006 & 31.19401 & 31.19406 & 26.33380 & 27.42028 & 27.42359 \\
\hline 127.00 & 36.91158 & 39.65579 & 39.65486 & 36.72393 & 36.83405 & 36.83640 \\
\hline 137.00 & 46.67034 & 48.63231 & 48.63285 & 46.68664 & 46.59745 & 46.59938 \\
\hline 147.00 & 56.57397 & 57.89847 & 57.89955 & 56.61703 & 56.45705 & 56.45879 \\
\hline 157.00 & 66.53723 & 67.45340 & 67.45432 & 66.59903 & 66.46595 & 66.46767 \\
\hline 167.00 & 76.52370 & 77.08904 & 77.08984 & 76.50332 & 76.39766 & 76.39940 \\
\hline 177.00 & 86.51886 & 86.88858 & 86.88931 & 86.48557 & 86.40846 & 86.41023 \\
\hline 187.00 & 96.51716 & 96.75424 & 96.75478 & 96.48843 & 96.43330 & 96.43508 \\
\hline 197.00 & 106.51657 & 106.59706 & 106.59728 & 106.40288 & 106.36023 & 106.36202 \\
\hline 207.00 & 116.51636 & 116.67091 & 116.67078 & 116.55261 & 116.52319 & 116.52499 \\
\hline 217.00 & 126.51629 & 126.48888 & 126.48906 & 126.39973 & 126.37558 & 126.37738 \\
\hline 227.00 & 136.51627 & 136.46008 & 136.46065 & 136.39791 & 136.37870 & 136.38049 \\
\hline 237.00 & 146.51626 & 146.57401 & 146.57489 & 146.53847 & 146.52419 & 146.52597 \\
\hline 247.00 & 156.51626 & 156.41475 & 156.41484 & 156.38607 & 156.37369 & 156.37545 \\
\hline 257.00 & 166.51626 & 166.40053 & 166.39979 & 166.37904 & 166.36868 & 166.37039 \\
\hline 267.00 & 176.51626 & 176.52571 & 176.52411 & 176.51173 & 176.50347 & 176.50515 \\
\hline & & & & & &
\end{tabular}

\section{Results and Discussions}

In this section, we choose the same parameters: $r=0.1, \sigma=0.2, K=100, T=1, \mu=0.05$, $\Delta t=0.01, a=0.02, M=0.01$, and $C=30$, as illustrated in the literature [46]. Then we calculate the call option values using the proposed schemes, described in previous section 4 , for different volatility models. We compare the approximate results with the exact value of the linear Black-Scholes model and among themselves also. 

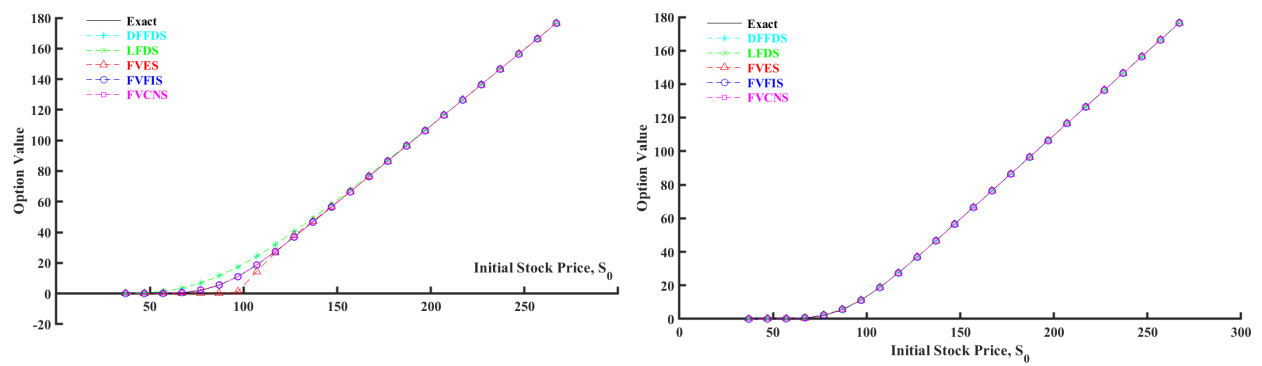

Figure 1. Approximate results of Equation (1) by using (a) Boyle and Vorst volatility model, and (b) Barles and Soner volatility model.

From Table 1 and Figure 8.7 (see Appendix), we observe that fully implicit FVS and CrankNicolson FVS provide comparatively better results than the other methods. Note that all of the methods provide poor results when the initial stock price is less than the strike price (here strike price, in comparison with the exact value of the linear Black-Scholes model.

From Table 8.3 in Appendix 8 and Figure 1 (a), we can make similar comments, but here the FVES gives a very poor approximation than the other methods when the initial stock price is less than the strike price $(K=100)$. Table 8.4 in Appendix 8 and Figure 1(b) show that all of the methods provide a closer approximation to the exact value of the linear Black-Scholes model for all of the initial stock price, whether it is greater than the strike price, $K=100$.

TABLE 2. Call option prices using RAPM volatility model.

\begin{tabular}{c|c|c|c|c|c|c}
\hline \multirow{2}{*}{$S_{0}$} & \multirow{2}{*}{$\begin{array}{c}\text { Exact } \\
\text { (Linear) }\end{array}$} & Finite Difference Schemes & \multicolumn{3}{|c}{ Finite Volume Schemes } \\
\cline { 3 - 7 } & DFFDS & LFDS & FVES & FVFIS & FVCNS \\
\hline 37.00 & 0.00001 & 0.09975 & 0.10198 & 0.00075 & 0.00054 & 0.00050 \\
\hline 47.00 & 0.00182 & 0.64320 & 0.64859 & 0.02029 & 0.01340 & 0.01297 \\
\hline 57.00 & 0.05078 & 2.01164 & 2.01581 & 0.16518 & 0.10771 & 0.10588 \\
\hline 67.00 & 0.45226 & 4.58788 & 4.59820 & 0.97606 & 0.65191 & 0.64795 \\
\hline 77.00 & 1.97686 & 8.35995 & 8.36458 & 3.28335 & 2.26632 & 2.26230 \\
\hline 87.00 & 5.46222 & 13.24558 & 13.25523 & 7.65193 & 5.63768 & 5.63688 \\
\hline 97.00 & 11.17037 & 19.14277 & 19.14296 & 13.90023 & 11.07370 & 11.07714 \\
\hline 107.00 & 18.71972 & 25.95302 & 25.96004 & 21.59995 & 18.66210 & 18.66616 \\
\hline 117.00 & 27.48006 & 33.49710 & 33.50138 & 30.05441 & 27.42028 & 27.42359 \\
\hline 127.00 & 36.91158 & 41.58132 & 41.58229 & 39.02457 & 36.83405 & 36.83640 \\
\hline 137.00 & 46.67034 & 50.16133 & 50.16454 & 48.32523 & 46.59745 & 46.59938 \\
\hline 147.00 & 56.57397 & 59.07941 & 59.08280 & 57.80665 & 56.45705 & 56.45879 \\
\hline 157.00 & 66.53723 & 68.31796 & 68.31997 & 67.49623 & 66.46595 & 66.46767 \\
\hline 167.00 & 76.52370 & 77.71942 & 77.72089 & 77.20105 & 76.39767 & 76.39941 \\
\hline 177.00 & 86.51886 & 87.32671 & 87.32812 & 87.03280 & 86.40846 & 86.41023 \\
\hline 187.00 & 96.51716 & 97.04281 & 97.04399 & 96.91429 & 96.43330 & 96.43509 \\
\hline 197.00 & 106.51657 & 106.79810 & 106.79881 & 106.75046 & 106.36023 & 106.36202 \\
\hline 207.00 & 116.51636 & 116.77932 & 116.77954 & 116.81690 & 116.52319 & 116.52498 \\
\hline 217.00 & 126.51629 & 126.56491 & 126.56478 & 126.62193 & 126.37555 & 126.37734 \\
\hline 227.00 & 136.51627 & 136.50685 & 136.50637 & 136.57895 & 136.37864 & 136.38041 \\
\hline 237.00 & 146.51626 & 146.59198 & 146.59115 & 146.67807 & 146.52410 & 146.52585 \\
\hline 247.00 & 156.51626 & 156.42589 & 156.42489 & 156.50633 & 156.37350 & 156.37521 \\
\hline 257.00 & 166.51626 & 166.40454 & 166.40336 & 166.47896 & 166.36838 & 166.37004 \\
\hline 267.00 & 176.51626 & 176.52231 & 176.52094 & 176.59035 & 176.50307 & 176.50468 \\
\hline & & & & & & \\
\hline
\end{tabular}


Finally, from Table 2 and the corresponding Figure 8.8 in Appendix 8, we may observe that for the RAPM volatility model, the FVCNS and FVFIS give better approximation than the other numerical schemes when the initial stock price is closer to and/or greater than the strike price. On the other hand, from Figures 2, 3, 4, it is clear that FVFIS and FVCNS produce comparatively better results than the other schemes for all of the volatility models. Figures 5, 6 depict the option prices at various time periods (from initial time $t=0$ to maturity time, $t=T$ ) with different initial stock values. The similar results of solution surface for option price by using Barles and Soner volatility model and RAPM volatility model are presented in Appendix 8, see Figures 8.9,8.10.
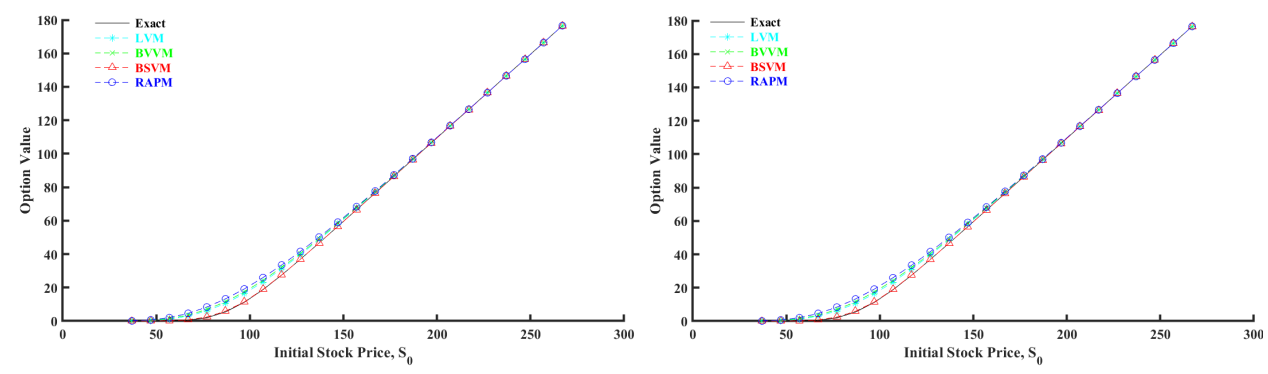

FIGURE 2. Approximate results of Equation (1) using (a) Dufort-Frankel Finite Difference Scheme, and (b) Laasonen Finite Difference Scheme.
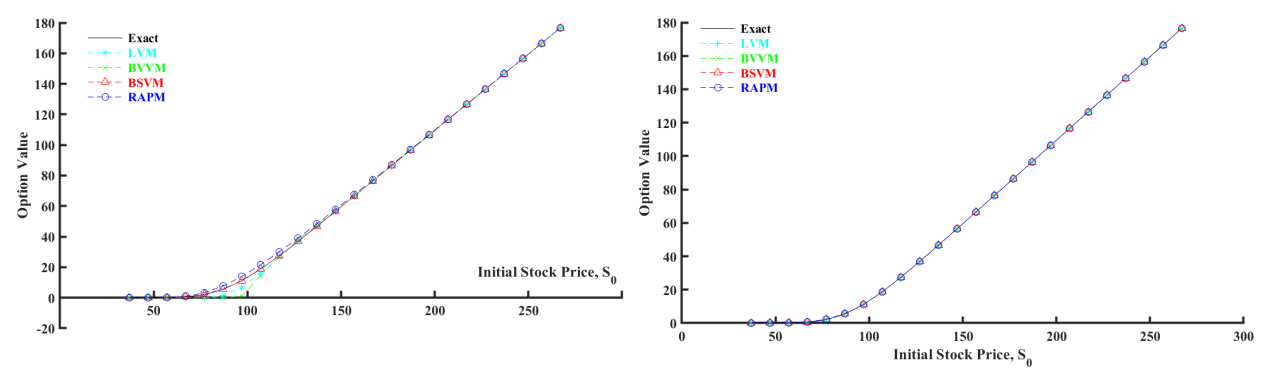

FIGURE 3. Approximate results of Equation (1) using (a) Finite Volume Explicit Scheme, and (b) Finite Volume Fully Implicit Scheme.

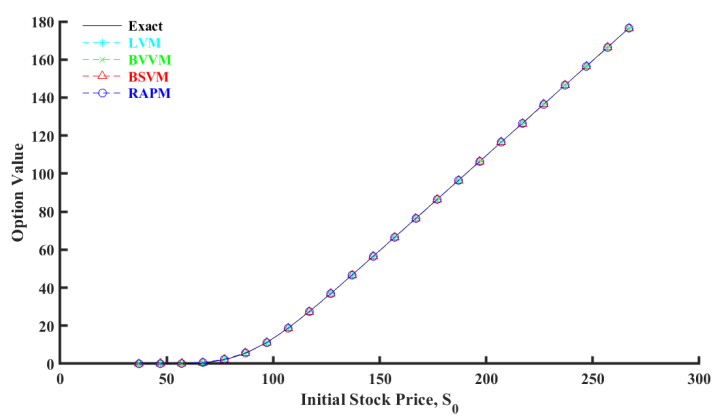

FIGURE 4. Approximate results of Equation (1) using Finite Volume Crank-Nicolson Scheme. 


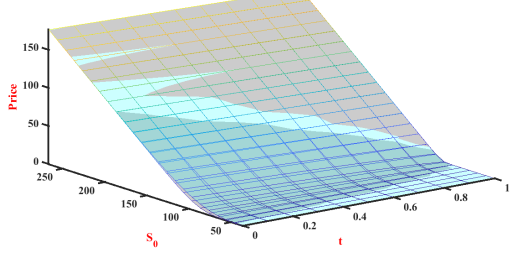

(A) LVM (DFFDS)

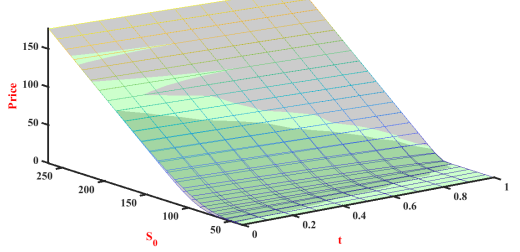

(в) LVM (LDS)

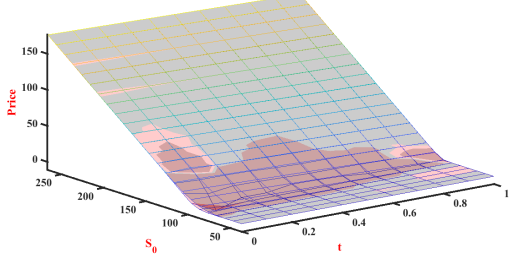

(c) LVM (FVES)

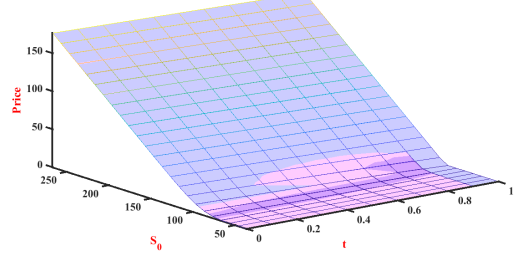

(D) LVM (FVCNS)

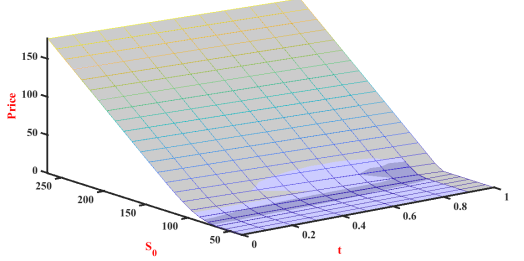

(E) LVM (FVFIS)

FIGURE 5. Solution surface for option price by using Leland volatility model.

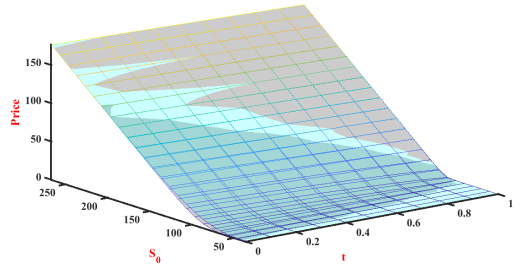

(A) BVVM (DFFDS)

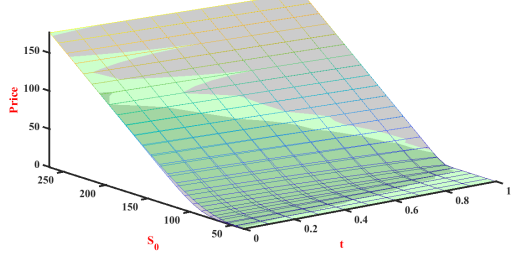

(B) BVVM (LDS)

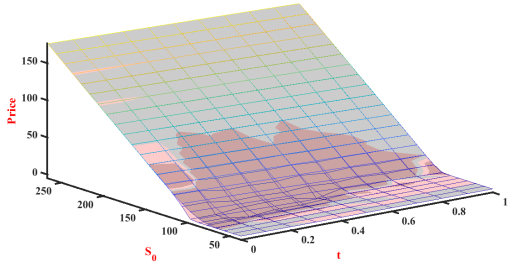

(c) BVVM (FVES)

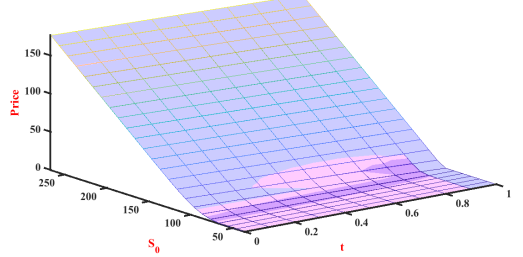

(D) BVVM (FVCNS)

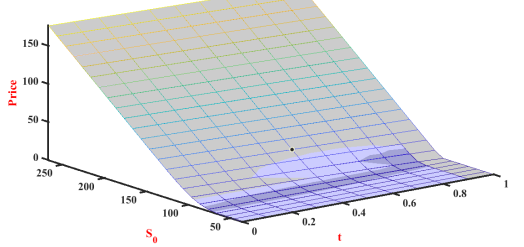

(E) BVVM (FVFIS)

FIGURE 6. Solution surface for option price by using Boyle and Vorst volatility model.

\section{CONCLUSION}

In this research work, we have derived some numerical schemes using the FVM and FDM to solve the non-linear Black-Scholes PDE for European option pricing with the transaction costs by exploiting the transformations available in the existing literature [46]. Thus we have modified the model equation accordingly to a non-linear parabolic PDE. For the convergence of these schemes, stability and consistency have been shown rigorously. Then these schemes have been applied to various volatility models. According to the visible results, as presented in the earlier sections, it 
is noted that all of the proposed schemes provide the best approximation to the exact value of the linear Black-Scholes model for all initial stock prices, regardless of whether they are closer to or greater than the strike price; particularly in the case of Barles and Soner Volatility Model. We may claim that the FVFIS and FVCNS approximate better than the other methods for all fourvolatility models. Thus, it is observed that the FVFIS and FVCNS are very effective and proficient in locating approximate solutions to non-linear Black-Scholes models. Notice that the limitation of these schemes is that they may offer poor results sometimes when the initial stock price is less than the strike price compared to the exact value of the linear Black-Scholes model. Finally, we may conclude that the proposed schemes may be applied to other non-linear partial differential equations to compute the numerical solutions with the desired accuracy.

Conflicts of Interest. The authors declare no competing interests exist.

Funding Statement. This research received no external funding.

\section{REFERENCES}

[1] J. C. Hull, Options, futures, and other derivatives, 8th ed., Pearson Prentice Hall, New Jersey, USA, 2009.

[2] N. Privault, Stochastic \& finance an introduction with market examples, 1st ed., CRC Press, 2013.

[3] J. Monique, Y. Marc, C. March, Mathematical methods for financial markets, Springer Science \& Business Media, 2009.

[4] J. R. Buchanan, An undergraduate introduction to financial mathematics, 3rd ed., World Scientific Publishing Company, 2012.

[5] A. Yves, P. Olivier, Computational methods for option pricing, Society for Industrial and Applied Mathematics, 2005.

[6] J. Guyon, P. Henry-Labordere, Nonlinear option pricing, CRC Press, 2014.

[7] F. Black, M. Scholes, The pricing of options and corporate liabilities, J. Polit. Econ. 81 (1973) 637-654. https: //www. jstor.org/stable/1831029.

[8] R. C. Merton, Theory of rational option pricing, Bell J. Econ. Manag. Sci. 4 (1973) 141-183. https://doi.org/ $10.2307 / 3003143$.

[9] M. M. Chawla, M. A. Al-Zanaidi, D. J. Evans, Generalized trapezoidal formulas for the Black-Scholes equation of option pricing, Int. J. Comput. Math. 80 (2003) 1521-1526. https://doi.org/10 .1080/00207160310001603299.

[10] A. S. Shinde, K. C. Takale, Study of Black-Scholes model and its applications, Procedia Eng. 38 (2012) $270-279$. http://dx.doi.org/10.1016/j.proeng.2012.06.035.

[11] L. Jódar, P. Sevilla-Peris, J. C. Cortés, R. Saha, A new direct method for solving the Black-Scholes equation, Appl. Math. Lett. 18 (2005) 29-32. http://dx.doi.org/10.1016/j.aml.2002.12.016.

[12] K. S. Patel, M. Mehra, High-order compact finite difference method for Black-Scholes PDE, Springer Proc. Math. Stat. 143 (2015) 393-403. 10.1007/978-81-322-2485-3_32.

[13] P. Roul, V. M. K. Prasad Goura, A sixth order numerical method and its convergence for generalized Black-Scholes PDE, J. Comput. Appl. Math. 377 (2020) 112881. https://doi.org/10.1016/j . cam. 2020.112881.

[14] M. Brenner, M. G. Subrahmanyam, A simple approach to option valuation and Hedging in the Black-Scholes model, Financ. Anal. J. 50 (1994) 25-28. https://www. jstor.org/stable/4479727.

[15] J. D. Macbeth, L. J. Merville, An empirical examination of the Black-Scholes call option pricing model, J. Finance, 34 (1979) 1173-1186. https://doi.org/10.2307/2327242. 
[16] J. Hok, T. L. R. Chan, Option pricing with Legendre polynomials, J. Comput. Appl. Math. 322 (2017) 25-45. http: //dx.doi.org/10.1016/j.cam.2017.03.027.

[17] M. S. Hossan, A. B. M. Hossain, M. S. Islam, Numerical solutions of Black-Scholes model by Du Fort-Frankel FDM and Galerkin WRM, Int. J. Math. Res. 9 (2020) 1-10. https://doi.org/10.18488/journal.24.2020.91.1.10.

[18] S. Alrabeei, M. Yousuf, Pricing European options under jump diffusion models with fast L-stable pade scheme, Int. J. Math. Comput. Sci. 14 (2020) 111-115.

[19] P. P. Boyle, Options: A Monte Carlo approach, J. Financ. Econ. 4 (1977) 323-338. https://doi.org/10.1016/ 0304-405X (77) $90005-8$.

[20] P. Carr, D. Madan, Option valuation using the fast fourier transform, J. Comput. Financ. 2 (1999) 61-73. https: //doi.org/10.21314/JCF.1999.043.

[21] P. Roul, V. M. K. Prasad Goura, A new higher order compact finite difference method for generalised Black-Scholes partial differential equation: European call option, J. Comput. Appl. Math. 363 (2020) 464-484. https://doi .org/ $10.1016 /$ j.cam.2019.06.015.

[22] S. Wang, A novel fitted finite volume method for the Black-Scholes equation governing option pricing, IMA J. Numer. Anal. 24 (2004) 699-720. https://doi.org/10.1093/imanum/24.4.699.

[23] F. Soleymani, A. Akgül, European option valuation under the Bates pide in finance: A numerical implementation of the Gaussian scheme, Discrete Cont. Dyn-S. 13 (2020) 889-909. http://dx. doi.org/10.3934/dcds. 2020052.

[24] Y. Fadaei, Z. A. Khan, A. Akgül, A greedy algorithm for partition of unity collocation method in pricing American options. Math. Methods Appl. Sci. 42 (2019) 5595-5606. https://doi.org/10.1002/mma. 5757.

[25] A. Q. M. Khaliq, B. Kleefeld, R. H. Liu, Solving complex PDE systems for pricing American options with regimeswitching by efficient exponential time differencing schemes, Numer. Methods Partial Differ. Equ. 29 (2013) 320-336. https://doi.org/10.1002/num.21714.

[26] A. Q. Khaliq, R. H. Liu, New numerical scheme for pricing American option with regime-switching, Int. J. Theor. Appl. Finance. 12 (2009) 319-340. https://doi.org/10.1142/S0219024909005245.

[27] M. Yousuf, High-order time-stepping scheme for pricing American option under Bates model, Int. J. Comput. Math. 96 (2019) 18-32. https://doi.org/10.1080/00207160.2017.1420785.

[28] M. Yousuf, A. Q. M. Khaliq, R. H. Liu, Pricing American options under multi-state regime switching with an efficient Lstable method, Int. J. Comput. Math. 92 (2015) 2530-2550. https: // doi .org/10 .1080/00207160.2015.1071799.

[29] P. Roul, A fourth order numerical method based on B-spline functions for pricing Asian options, Comput. Math. with Appl. 80 (2020) 504-521. https://doi.org/10.1016/j.camwa.2020.04.001.

[30] K. S. Patel, M. Mehra, High-order compact finite difference scheme for pricing Asian option with moving boundary condition, Differ. Equations Dyn. Syst. 27 (2019) 39-56. https://doi.org/10.1007/s12591-017-0372-8.

[31] B. A. Wade, A. Q. M. Khaliq, M. Yousuf, J. Vigo-Aguiar, R. Deininger, On smoothing of the Crank-Nicolson scheme and higher-order schemes for pricing barrier options, J. Comput. Appl. Math. 204 (2007) 144-158. https://doi. org/10.1016/j.cam.2006.04.034.

[32] M. H. Akrami, G. H. Erjaee, Numerical solutions for fractional Black-Scholes option pricing equation, Glob. Anal. Discret. Math. 1 (2016) 9-14. https://doi.org/10.1016/j.camwa.2016.02.007.

[33] S. Kumar, A. Yildirim, Y. Khan, H. Jafari, K. Sayevand, L. Wei, Analytical solution of fractional Black-Scholes European option pricing equation by using Laplace transform, J. Fract. Calc. Appl. 2 (2012) 1-9.

[34] M. H. Akrami, G. H. Erjaee, Examples of analytical solutions by means of Mittag-Leffler function of fractional Black-Scholes option pricing equation, Fract. Calc. Appl. Anal. 18 (2015) 38-47. https://doi.org/10.1515/ fca-2015-0004.

[35] P. Ehrhardt, A. Unterreiter, The numerical solution of nonlinear Black-Scholes equations, Technische Universitat Berlin, 28 (2008). 
[36] H. Soner, S. Shreve, J. Cvitanic, There is no nontrivial hedging portfolio for option pricing with transaction costs, Ann. Appl. Probab. 5 (1995) 5327-355. https://www. jstor.org/stable/2245301.

[37] H. E. Leland, Option pricing and replication with transactions costs, J. Finance. 40 (1985) 1283-1301. https: //doi.org/10.1111/j.1540-6261.1985.tb02383.x.

[38] P. P. Boyle, T. Vorst, Option replication in discrete time with transaction costs, J. Finance. 47 (1992) $271-293$. https://doi.org/10.1111/j.1540-6261.1992.tb03986.x.

[39] J. Dewynne, A. Whalley, P. Wilmott, Path-dependent options and transaction costs, Phil. Trans. R. Soc. Lond. A. 347 (1994) 517-529. https://www. jstor.org/stable/54362.

[40] A. E. Whalley, P. Wilmott, An asymptotic analysis of an optimal hedging model for option pricing with transaction costs, Math. Financ. 7 (1997) 307-324. https://doi.org/10.1111/1467-9965.00034.

[41] M. Monoyios, Option pricing with transaction costs using a Markov chain approximation, J. Econ. Dyn. Control. 28 (2004) 889-913. https://doi.org/10.1016/S0165-1889(03)00059-9.

[42] R. Company, E. Navarro, J. Ramón Pintos, E. Ponsoda, Numerical solution of linear and nonlinear Black-Scholes option pricing equations, Comput. Math. with Appl. 56 (2008) 813-821. https://doi.org/10.1016/j.camwa. 2008.02 .010$.

[43] G. Barles, H. M. Soner, Option pricing with transaction costs and a nonlinear Black-Scholes equation, Financ. Stochastic. 2 (1998) 369-397. https://doi.org/10.1007/s007800050046.

[44] M. Yousuf, A. Q. M. Khaliq, B. Kleefeld, The numerical approximation of nonlinear black-scholes model for exotic path-dependent American options with transaction cost, Int. J. Comput. Math. 89 (2012) 1239-1254. https://doi. org/10.1080/00207160.2012.688115.

[45] D. C. Lesmana, S. Wang, An upwind finite difference method for a nonlinear Black-Scholes equation governing European option valuation under transaction costs, Appl. Math. Comput. 219 (2013) 8811-8828. https://doi. org/10.1016/j.amc.2012.12.077.

[46] J. Ankudinova, M. Ehrhardt, On the numerical solution of nonlinear Black-Scholes equations, Comput. Math. with Appl. 56 (2008) 799-812. https://doi.org/10.1016/j. camwa.2008.02.005.

[47] R. L. Valkov, Fitted strong stability-preserving schemes for the Black-Scholes Barenblatt equation, Int. J. Comput. Math. 92 (2015) 2475-2497. https://doi.org/10.1080/00207160.2015.1069818.

[48] R. Valkov, Predictor-corrector balance method for the worst-case 1D option pricing, Comput. Methods Appl. Math. 16 (2016) 175-186. https://doi.org/10.1515/cmam-2015-0029.

[49] P. Heider, Numerical methods for non-linear Black-Scholes equations, Appl. Math. Finance. 17 (2010) $59-81$. https://doi.org/10.1080/13504860903075670.

[50] E. Dremkova, M. Ehrhardt, A high-order compact method for nonlinear Black-Scholes option pricing equations of American options, Int. J. Comput. Math. 88 (2011) 2782-2797. https://doi.org/10.1080/00207160.2011. 558574.

[51] N. Ishimura, Remarks on the nonlinear Black-Scholes equations with the effect of transaction costs, Asia-Pac. Fin. Mark. 17 (2010) 241-259. https://doi.org/10.1007/s10690-010-9115-3.

[52] S. D. Hodges, A. Neuberger, Optimal replication of contingent claims under transactions costs, Rev. Futures Mark. 8 (1989) 222-239.

[53] M. Kratka, No Mystery Behind the Smile. Risk-London-Risk magazine limited, 11 (1998) 67-71.

[54] M. Jandačka, D. Ševčovič, On the risk-adjusted pricing-methodology-based valuation of vanilla options and explanation of the volatility smile, J. Appl. Math. 2005 235-258. https://doi .org/10.1155/JAM. 2005.235.

[55] K. A. Hoffmann, S. T. Chiang, Computational fluid dynamics Volume I, Engineering Education System, 2000.

[56] W. Malalasekera, H. K. Versteeg, An introduction to computational fluid dynamics: the finite volume method, Pearson education, 2007. 


\section{Appendix}

This section contains the supporting figures and tables to observe the accuracy of the solution methodologies.

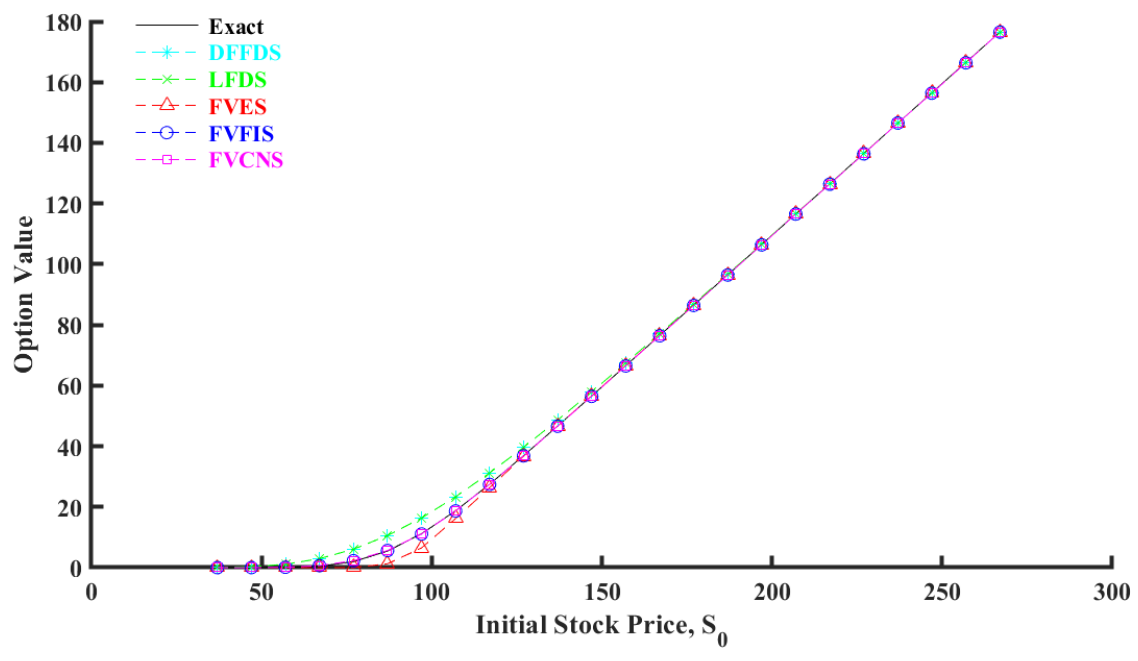

FIGURE 8.7. Approximate results of Equation (1) by using Leland volatility model.

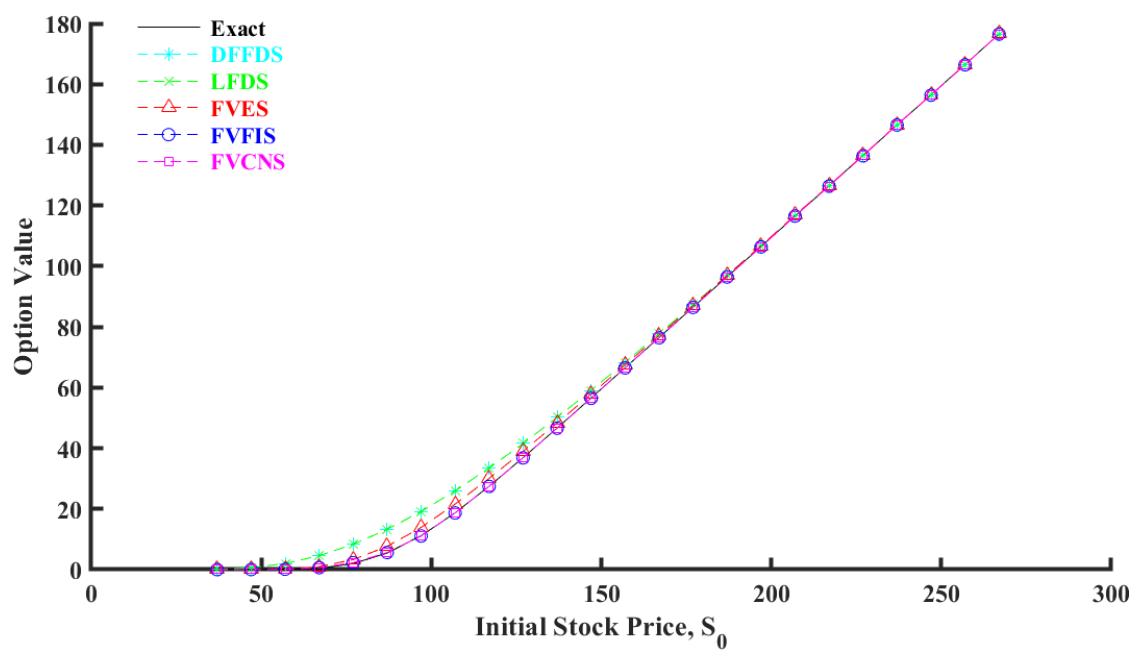

FIGURE 8.8. Approximate results of Equation (1) by using RAPM volatility model. 
TABLE 8.3. Call option prices using Boyle and Vorst volatility model.

\begin{tabular}{c|c|c|c|c|c|c}
\hline \multirow{2}{*}{$S_{0}$} & \multirow{2}{*}{$\begin{array}{c}\text { Exact } \\
\text { (Linear) }\end{array}$} & \multicolumn{2}{|c|}{ Finite Difference Schemes } & \multicolumn{3}{|c}{ Finite Volume Schemes } \\
\cline { 3 - 7 } & DFFDS & LFDS & FVES & FVFIS & FVCNS \\
\hline 37.00 & 0.00001 & 0.08130 & 0.08362 & 0.00000 & 0.00054 & 0.00050 \\
\hline 47.00 & 0.00182 & 0.44919 & 0.45475 & 0.00016 & 0.01340 & 0.01297 \\
\hline 57.00 & 0.05078 & 1.43187 & 1.43827 & 0.00137 & 0.10771 & 0.10588 \\
\hline 67.00 & 0.45226 & 3.52436 & 3.53389 & 0.00181 & 0.65191 & 0.64795 \\
\hline 77.00 & 1.97686 & 6.88032 & 6.88512 & -0.01095 & 2.26632 & 2.26230 \\
\hline 87.00 & 5.46222 & 11.52515 & 11.53065 & -0.03314 & 5.63768 & 5.63688 \\
\hline 97.00 & 11.17037 & 17.36380 & 17.36252 & 1.18226 & 11.07370 & 11.07714 \\
\hline 107.00 & 18.71972 & 24.30605 & 24.30805 & 14.49730 & 18.66210 & 18.66616 \\
\hline 117.00 & 27.48006 & 32.07120 & 32.07189 & 26.66264 & 27.42028 & 27.42359 \\
\hline 127.00 & 36.91158 & 40.41514 & 40.41449 & 37.98090 & 36.83405 & 36.83640 \\
\hline 137.00 & 46.67034 & 49.26375 & 49.26507 & 47.25031 & 46.59745 & 46.59938 \\
\hline 147.00 & 56.57397 & 58.41457 & 58.41649 & 56.80881 & 56.45705 & 56.45879 \\
\hline 157.00 & 66.53723 & 67.86188 & 67.86337 & 66.66025 & 66.46595 & 66.46767 \\
\hline 167.00 & 76.52370 & 77.41353 & 77.41510 & 76.50821 & 76.39767 & 76.39940 \\
\hline 177.00 & 86.51886 & 87.14056 & 87.14251 & 86.47640 & 86.40846 & 86.41023 \\
\hline 187.00 & 96.51716 & 96.94695 & 96.94884 & 96.47214 & 96.43330 & 96.43509 \\
\hline 197.00 & 106.51657 & 106.75019 & 106.75144 & 106.38908 & 106.36023 & 106.36202 \\
\hline 207.00 & 116.51636 & 116.78198 & 116.78255 & 116.54146 & 116.52319 & 116.52500 \\
\hline 217.00 & 126.51629 & 126.57938 & 126.58055 & 126.39087 & 126.37558 & 126.37738 \\
\hline 227.00 & 136.51627 & 136.53059 & 136.53250 & 136.39143 & 136.37869 & 136.38048 \\
\hline 237.00 & 146.51626 & 146.62463 & 146.62715 & 146.53436 & 146.52418 & 146.52596 \\
\hline 247.00 & 156.51626 & 156.45839 & 156.45983 & 156.38285 & 156.37367 & 156.37541 \\
\hline 257.00 & 166.51626 & 166.43684 & 166.43715 & 166.37675 & 166.36863 & 166.37034 \\
\hline 267.00 & 176.51626 & 176.55434 & 176.55347 & 176.51042 & 176.50341 & 176.50508 \\
\hline & & & & & & \\
\end{tabular}

TABLE 8.4. Call option prices using Barles and Soner volatility model.

\begin{tabular}{c|c|c|c|c|c|c}
\hline \multirow{2}{*}{$S_{0}$} & \multirow{2}{*}{$\begin{array}{c}\text { Exact } \\
\text { Linear) }\end{array}$} & \multicolumn{2}{|l|}{ Finite Difference Schemes } & \multicolumn{3}{|c}{ Finite Volume Schemes } \\
\cline { 3 - 7 } & DFFDS & LFDS & FVES & FVFIS & FVCNS \\
\hline 37.00 & 0.00001 & 0.00056 & 0.00060 & 0.00047 & 0.00054 & 0.00050 \\
\hline 47.00 & 0.00182 & 0.01448 & 0.01496 & 0.01255 & 0.01340 & 0.01297 \\
\hline 57.00 & 0.05078 & 0.11766 & 0.11964 & 0.10402 & 0.10771 & 0.10588 \\
\hline 67.00 & 0.45226 & 0.70853 & 0.71289 & 0.64387 & 0.65191 & 0.64795 \\
\hline 77.00 & 1.97686 & 2.42414 & 2.42846 & 2.25831 & 2.26632 & 2.26230 \\
\hline 87.00 & 5.46222 & 5.90671 & 5.90840 & 5.63756 & 5.63768 & 5.63688 \\
\hline 97.00 & 11.17037 & 11.38951 & 11.38681 & 11.08596 & 11.07370 & 11.07714 \\
\hline 107.00 & 18.71972 & 18.90018 & 18.89694 & 18.68219 & 18.66210 & 18.66616 \\
\hline 117.00 & 27.48006 & 27.57177 & 27.56903 & 27.44285 & 27.42028 & 27.42359 \\
\hline 127.00 & 36.91158 & 36.90926 & 36.90718 & 36.85630 & 36.83405 & 36.83640 \\
\hline 137.00 & 46.67034 & 46.63600 & 46.63420 & 46.61611 & 46.59745 & 46.59938 \\
\hline 147.00 & 56.57397 & 56.47483 & 56.47316 & 56.47209 & 56.45705 & 56.45879 \\
\hline 157.00 & 66.53723 & 66.47486 & 66.47318 & 66.47729 & 66.46595 & 66.46767 \\
\hline 167.00 & 76.52370 & 76.40243 & 76.40071 & 76.40662 & 76.39766 & 76.39940 \\
\hline 177.00 & 86.51886 & 86.41173 & 86.40997 & 86.41573 & 86.40846 & 86.41022 \\
\hline 187.00 & 96.51716 & 96.43561 & 96.43381 & 96.43938 & 96.43329 & 96.43508 \\
\hline 197.00 & 106.51657 & 106.36234 & 106.36053 & 106.36582 & 106.36022 & 106.36202 \\
\hline 207.00 & 116.51636 & 116.52509 & 116.52327 & 116.52827 & 116.52319 & 116.52499 \\
\hline 217.00 & 126.51629 & 126.37745 & 126.37563 & 126.38047 & 126.37557 & 126.37737 \\
\hline 227.00 & 136.51627 & 136.38055 & 136.37873 & 136.38340 & 136.37869 & 136.38048 \\
\hline 237.00 & 146.51626 & 146.52603 & 146.52421 & 146.52870 & 146.52418 & 146.52596 \\
\hline 247.00 & 156.51626 & 156.37561 & 156.37380 & 156.37798 & 156.37368 & 156.37543 \\
\hline 257.00 & 166.51626 & 166.37066 & 166.36886 & 166.37271 & 166.36866 & 166.37038 \\
\hline 267.00 & 176.51626 & 176.50552 & 176.50374 & 176.50725 & 176.50346 & 176.50514 \\
\hline & & & & & &
\end{tabular}




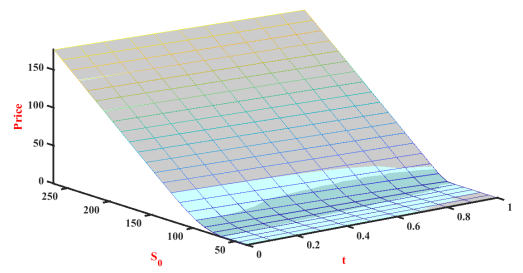

(A) BSVM (DFFDS)

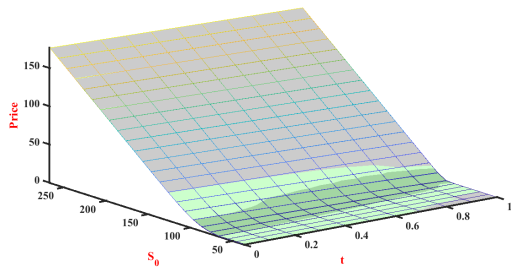

(в) BSVM (LDS)

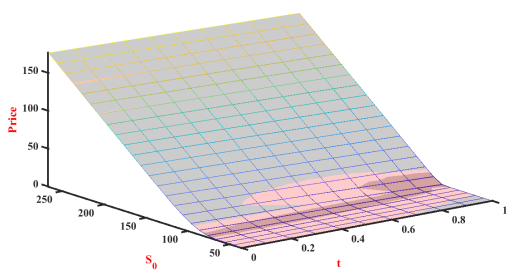

(c) BSVM (FVES)

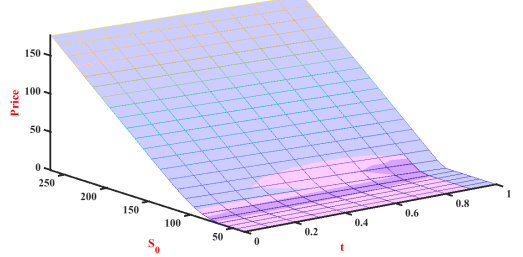

(D) BSVM (FVCNS)

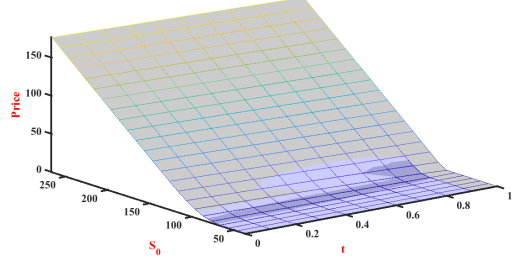

(E) BSVM (FVFIS)

FIGURE 8.9. Solution surface for option price by using Barles and Soner volatility model.

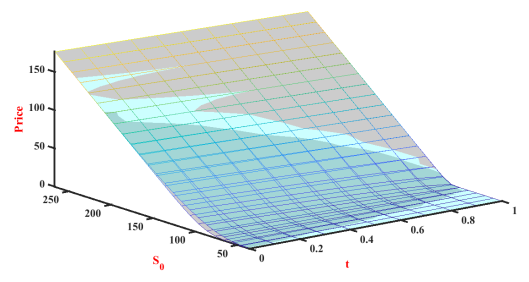

(A) RAPM (DFFDS)

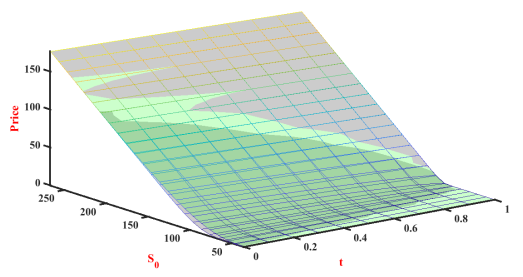

(B) RAPM (LDS)

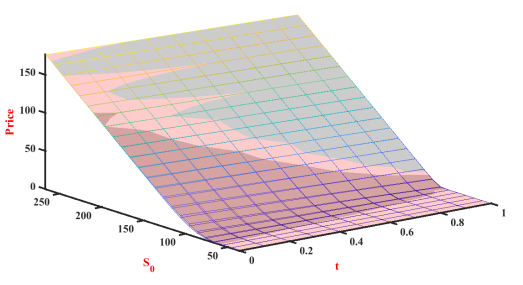

(c) RAPM (FVES)

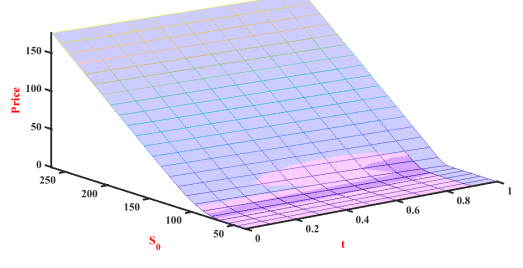

(D) RAPM (FVCNS)

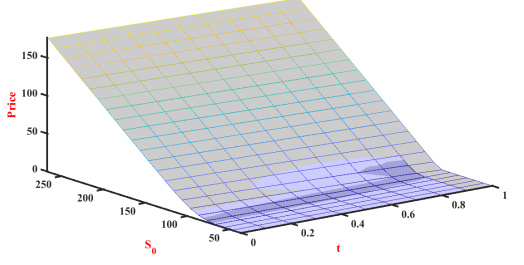

(E) RAPM (FVFIS)

FIGURE 8.10. Solution surface for option price by using RAPM volatility model. 